\title{
Curvature Effect of a Non-Power-Law Spectrum and Spectral Evolution of GRB X-Ray Tails
}

\author{
Bin-Bin Zhang ${ }^{1}$, Bing Zhang ${ }^{1}$, En-Wei Liang ${ }^{2}$, Xiang-Yu Wang ${ }^{3}$
}

\begin{abstract}
The apparent spectral evolution observed in the steep decay phase of many GRB early afterglows raises a great concern of the high-latitude "curvature effect" interpretation of this phase. However, previous curvature effect models only invoked a simple power law spectrum upon the cessation of the prompt internal emission. We investigate a model that invokes the "curvature effect" of a more general non-power-law spectrum and test this model with the Swift/XRT data of some GRBs. By comparing the simulated lightcurves/spectra with the observed ones, we show that one can reproduce both the observed lightcurve and the apparent spectral evolution of several GRBs using a model invoking a cut-off power-law spectrum. GRB 050814 is presented as an example. This suggests that at least for some GRBs, the fast spectral evolution in the steep-decay phase can be explained by the curvature effect invoking a non-power-law spectrum.
\end{abstract}

Subject headings: gamma-rays: bursts

\section{INTRODUCTION}

The rapid autonomous slewing capabilities of Swift enable the monitoring of GRB X-ray afterglows right after or even during the prompt gamma-ray emission (Gehrels et al. 2004). Most of those early X-Ray afterglows show a steep decay phase around 100 1000 seconds after the burst trigger (Tagliaferri et al. 2005; Zhang et al. 2006; Nousek et al 2006). The main characteristics of this steep decay phase include the following. (1) It connects smoothly to the prompt $\gamma$-ray light curve extrapolated to the X-ray band, suggesting that it is the "tail" of the prompt emission (Barthelmy et al. 2005, O'Brien et 2006, Liang et al

\footnotetext{
${ }^{1}$ Department of Physics and Astronomy, University of Nevada, Las Vegas, NV 89154, USA; zbb,bzhang@physics.unlv.edu

${ }^{2}$ Department of Physics, Guangxi University, Nanning 530004, China

${ }^{3}$ Department of Astronomy, Nanjing University, Nanjing 210093, China
} 
2006). (2) The decay slope is typically $3 \sim 5$ when choosing the GRB trigger time as the zero time point $t_{0}$ (Tagliaferri et al. 2005; Nousek et al. 2006; Zhang et al 2006). (3) The time-averaged spectral index of steep decay phase is much different from that of the later shallow decay phase, indicating that it is a distinct new component that is unrelated to the conventional afterglow components (Zhang et al 2006; Liang et al. 2007). (4) Strong spectral evolution exists in about one third of the bursts that have a steep decay phase (Zhang et al. 2007, hereafter ZLZ07; Butler \& Kocevski 2007; Starling et al. 2008). All these features suggest that the steep decay phase holds the key to understand the connection between the prompt emission (internal) phase and the traditional afterglow (external) phase. Any proposed model (see Mészáros 2006; Zhang 2007 for reviews) should be able to explain these features.

The so called "curvature effect", which accounts for the delayed photon emission from high latitudes with respect to the line of sight upon the abrupt cessation of emission in the prompt emission region (Fenimore et al. 1996; Kumar \& Panaitescu 2000; Dermer 2004; Dyks et al. 2005; Qin 2008a), has been suggested to play an important role in shaping the sharp flux decline in GRB tails (Zhang et al. 2006; Liang et al. 2006; Wu et al. 2006). In the simplest model, it is assumed that the high-latitude locations have exactly the same physical conditions as the one along the line of sight, and that the instantaneous spectrum at the end of the prompt emission has a simple power law spectrum with a spectral index $\beta$. The predicted temporal decay index of the emission is (with the convention $F_{\nu} \propto t^{-\alpha} \nu^{-\beta}$ )

$$
\alpha=2+\beta
$$

if $t_{0}$ is taken as the beginning of the last emission episode before the cessation of emission. Liang et al. (2006) have tested this prediction against the data and found that the curvature effect interpretation is generally valid. However, they adopted time-averaged $\beta$ values and did not analyze the time-dependent X-ray spectra in detail. The strong spectral evolution identified in a group of GRB tails (ZLZ07) apparently violate Eq.(1), which is valid only for a constant $\beta$. ZLZ07 then investigated a curvature effect model by assuming a structured jet with varying $\beta$ at different latitudes and that the line of sight is near the jet axis. 1 . One would then expect that Eq.(11) is roughly satisfied, with both $\alpha$ and $\beta$ being time-dependent. ZLZ07 found that this model does not fit the data well.

These facts do not rule out the curvature effect interpretation of GRB tails, however. This is because the instantaneous spectrum upon the cessation of prompt emission may well be in a more complicated form than power-law, such as a Band-function or a power law with

\footnotetext{
${ }^{1}$ Notice that this structured jet model is different from the traditional one that invokes an angle-dependent energy/Lorentz factor, but not spectral index (Zhang \& Mészáros 2002; Rossi et al. 2002).
} 
an exponential cut-off. If this is the case, as the emission from progressively higher latitudes reach the observer, the XRT band is sampling different segments of the intrinsic spectrum (Fig.1). This would introduce an apparent spectral evolution in the decaying tail. The main goal of this paper is to test this more general curvature effect model using the available Swift XRT data.

\section{Curvature Effect of a Non-powerlaw Spectrum}

We consider a general non-power-law spectrum in the form of

$$
F_{\nu}(\nu)=F_{\nu, c} G(\nu)
$$

where $G(\nu)$ is the function form of the spectrum with a characteristic frequency $\nu_{c}$ so that $G\left(\nu_{c}\right)=1$, and $F_{\nu, c}=F_{\nu}\left(\nu_{c}\right)$ is the normalization of the spectrum at $\nu=\nu_{c}$.

The curvature effect states that given a same spectrum at different latitudes with respect to the line of sight, one has $F_{\nu, c} \propto \mathcal{D}^{2}$ and $\nu_{c} \propto \mathcal{D}$, where $\mathcal{D}$ is the Doppler factor. If the high-latitude angle $\theta \gg \Gamma$, the Dopper factor $\mathcal{D} \propto t^{-1}$, so that $F_{\nu, c} \propto t^{-2}, \nu_{c} \propto t^{-1}$ (Kumar \& Panaitescu 2000). Considering the $t_{0}$ effect (Zhang et al. 2006; Liang et al. 2006), this can be written a: 2

$$
F_{\nu, c}(t)=F_{\nu, c, p}\left(\frac{t-t_{0}}{t_{p}-t_{0}}\right)^{-2}
$$

and

$$
\nu_{c}(t)=\nu_{c, p}\left(\frac{t-t_{0}}{t_{p}-t_{0}}\right)^{-1}
$$

for $t \gg t_{p}$, where $t_{p}$ is the epoch when the curvature-effect decay starts (or the "peak" time of the lightcurve), $F_{\nu, c, p}=F_{\nu, c}\left(t_{p}\right)$ and $\nu_{c, p}=\nu_{c}\left(t_{p}\right)$. Notice that in the case of $G(\nu)=\left(\nu / \nu_{c}\right)^{-\beta}$ (a pure power law spectrum), one derives $F_{\nu} \propto\left(t-t_{0}\right)^{-\beta-2}$. This is the relation Eq.(11). Eqs. (3) and (41) can describe the curvature effect evolution of a more general form of spectrum $G(\nu)$.

We consider several physically motivated non-powerlaw spectra with a characteristic frequency $\nu_{c}$, including the cut-off power law spectrum and the Band-function (Band et al. 1993). To explore the compatibility with the data, we also investigate different forms of

\footnotetext{
${ }^{2}$ Notice that the denominator in Eq.(3) is $\left(t_{p}-t_{0}\right)$ rather than $t_{0}$ used in our previous works (Zhang et al. 2006; Liang et al. 2007; ZLZ07). This gives a precise physical meaning of the coefficient in the curvature effect model. Using the previous notation gives a slightly different coefficient that is related to $F_{\nu, c, p}$ through a constant factor.
} 


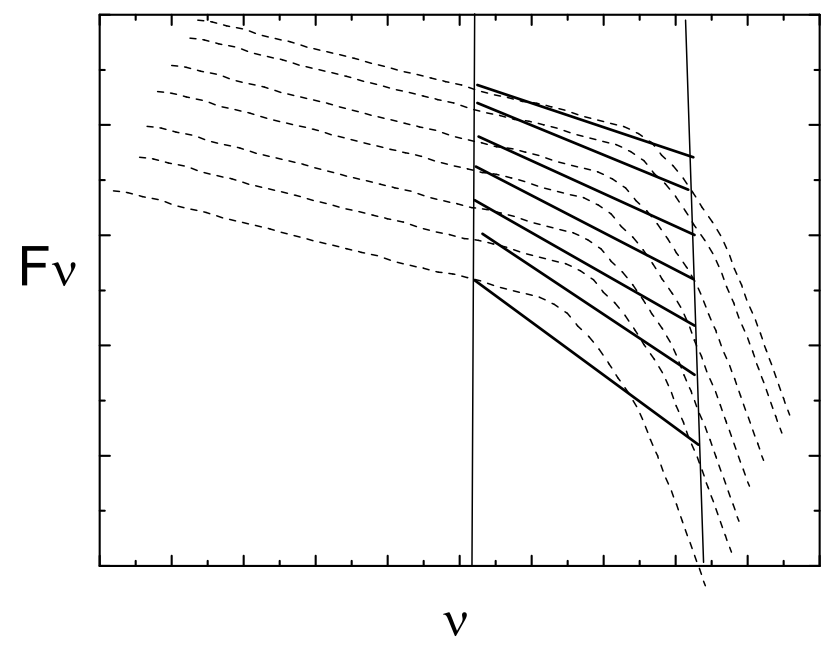

Fig. 1.- A schematic picture showing that shifting a set of non-power-law spectra in time can equivalently give an apparent spectral evolution in a fixed band. The dashed lines represent a set of exponential-like spectra, whose $F_{\nu_{p}}(t)$ and $\nu_{p}(t)$ drop down with time according to Eqs. (3) and (44). The two vertical solid lines bracket the observed energy band. The thick solid lines denote the effective power law fits to the time-dependent spectra at each time step. 
the cutoffs with varying sharpness. In all cases, the $F_{\nu_{p}}(t)$ and $\nu_{p}(t)$ follow Eqs.(3) and (44). When $\nu_{c}(t)$ drops across an observational narrow energy band, e.g. the Swift/XRT band, it introduces an apparent spectral softening with time, which, if fitted by a power law, showing an increase of photon index with time.

In the meantime, the flux within the observing band drops down rapidly, leading to an apparent steep decay phase in the lightcurve. This scenario is illustrated in Fig.1.

\section{Data Reduction and Simulation Method}

We consider a time-dependent cutoff power law photon spectrum taking the form of

$$
N(E, t)=N_{0}(t)\left(\frac{E}{1 \mathrm{keV}}\right)^{-\Gamma} \exp \left[-\left(\frac{E}{E_{c}(t)}\right)^{k}\right]
$$

where $\Gamma=\beta+1$ is the power law photon index, and $k$ is a parameter to define the sharpness of the high energy cutoff in the spectrum, $E_{c}(t)$ is the time-dependent characteristic photon energy, and $N_{0}(t)$ is a time-dependent photon flux (in units of photons $\cdot \mathrm{keV}^{-1} \mathrm{~cm}^{-2} \mathrm{~s}^{-1}$ ) at 1 $\mathrm{keV}$ (Arnaud 1996). The choice of this function was encouraged by the fact that the spectral evolution of some GRB tails can be fitted by such an empirical model (Campana et al. 2006; ZLZ07; Yonetoku et al. 2008). According to Eqs.(3) and (44), and noticing the conversion between the photon flux and the emission flux density, i.e. $F_{\nu} \propto E N(E)$, we get

$$
N\left(E_{c}, t\right)=N_{c, p}\left(\frac{t-t_{0}}{t_{p}-t_{0}}\right)^{-1}
$$

and

$$
E_{c}(t)=E_{c, p}\left(\frac{t-t_{0}}{t_{p}-t_{0}}\right)^{-1}
$$

where $N_{c, p}=N\left(E_{c}, t_{p}\right)$, and $E_{c, p}=E_{c}\left(t_{p}\right)$. This gives

$$
N_{0}(t)=N_{0, p}\left(\frac{t-t_{0}}{t_{p}-t_{0}}\right)^{-(1+\Gamma)} .
$$

We therefore have five parameters altogether, namely, $N_{0, p}, E_{c, p}, \Gamma, t_{0}$, and $k$. Notice that $t_{p}$ is not a free parameter, it is the beginning of the steep decay, which could be read off from the lightcurve. In case of an observational gap, $t_{p}$ can be roughly estimated as the end of the prompt emission. At any time $t$, the model spectrum can be determined once these parameters are given. One can then confront the model with the real GRB data. The procedure includes the following steps. 


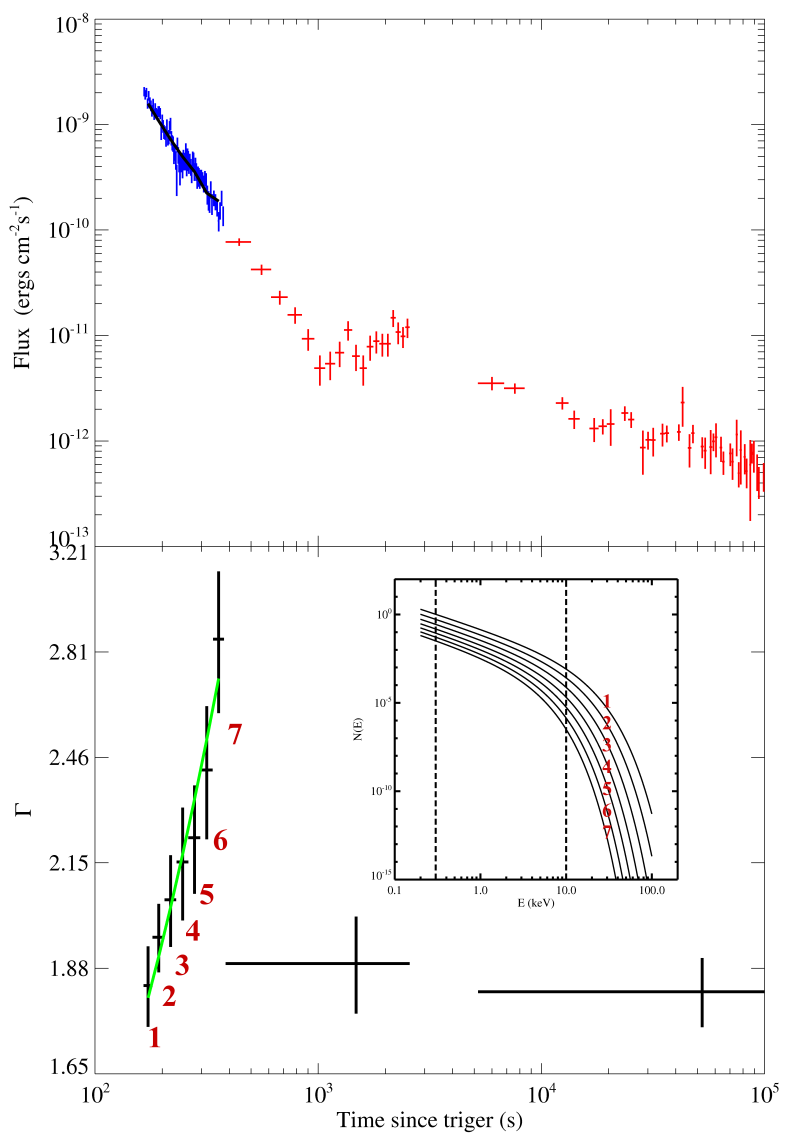

Fig. 2.- The lightcurve (upper panel) and spectral evolution (lower panel) of the X-ray tail of GRB 050814 with the best-fit theoretical model (black curve in upper panel and green curve in lower panel). The blue and red data points are the window timing and photon counting data, respectively. The inset shows time-dependent theoretical spectra with the XRT band $(0.3-10 \mathrm{keV})$ bracketed by two vertical lines. The integers denote the time segments for the time-resolved spectral analysis. 

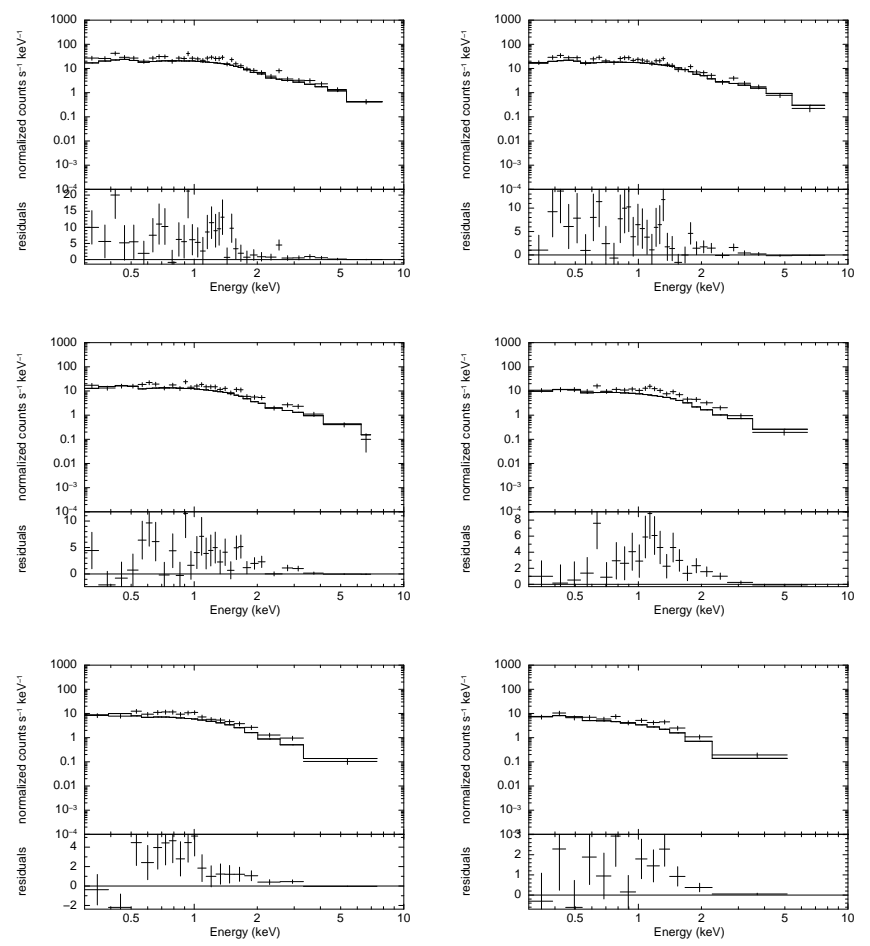

Fig. 3.- Simulated time-dependent spectra of GRB050814 with the best-fit parameters. The time intervals for each panel (from top left to bottom right) are 1, 3, 4, 5, 6,7 repectively as in Fig. 2. In each panel, the data histogram displays the simulated spectrum, and the solid line displays the best-fit power law model (wabs*zwasb*powerlaw in XSPEC) that is used to derive the time-dependent photon index $\Gamma$. 

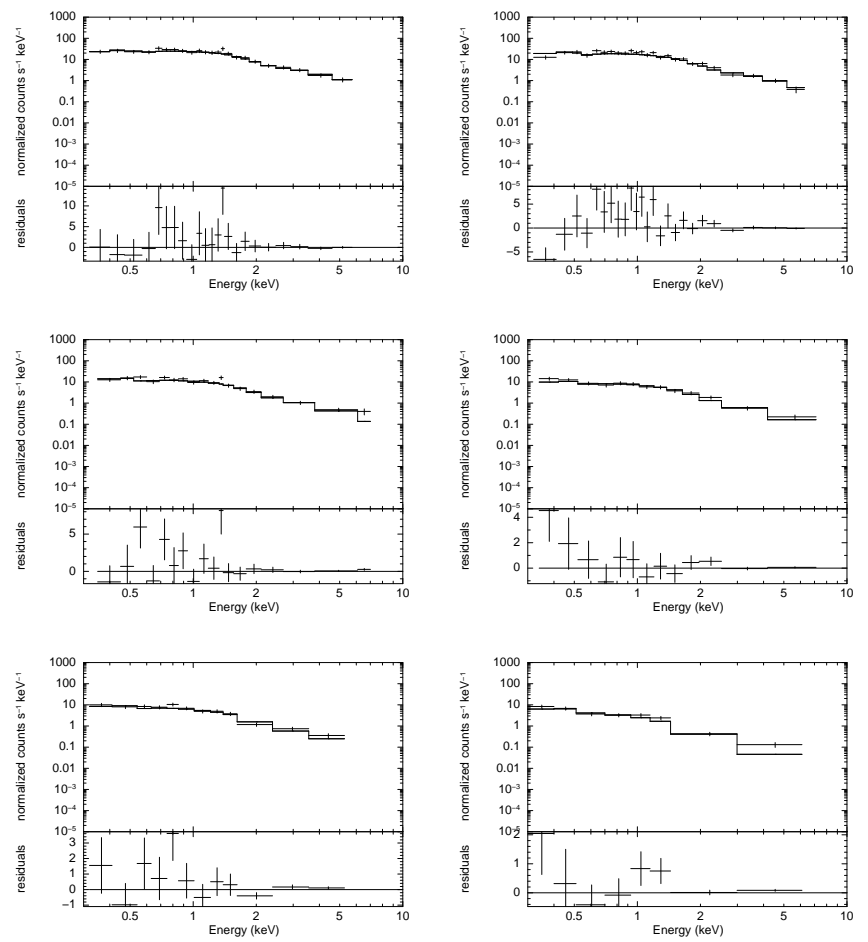

Fig. 4.- Observed time-dependent spectra of GRB050814. The data histogram displays the observed spectrum. Other symbols and time intervals in each panel are the same as in Fig.3. 
(1) For a given burst, we extract its Swift/XRT light curve and $n$ slices of timedependent spectra using the standard HEASoft/Swift Package. The details of the data reduction method were described in ZLZ07.

(2) Given a trial set of parameters in the theoretical spectra $\left.3 N_{0, p}, E_{c, p}, \Gamma, t_{0}\right\}$, using Eqs. (5. -7) we model $n$ time-dependent theoretical spectra that correspond to the time bins that are used to derive the time-dependent observed spectra.

(3) Based on the theoretical spectra of each time slice, we simulate the corresponding model spectra by taking account of the observational effects, including the Swift/XRT response matrix, the absorption column densities $\left(N_{H}\right)$ of both the Milky Way (extracted from the observations from step 1) and the host galaxy of the burst (a free parameter), the redshift (if applicable), and a Poisson noise background. Notice that $n_{H, h o s t}$ is another parameter introduced in the model spectra (besides the other parameters introduced in the theoretical spectra). All these faked spectra can be obtained using HEASoft (Version 6.4) and Xspec (Version 12.4)

(4) We fit the faked model spectra with a simple power law model, i.e. wabs *wabs* powerlaw (or wabs * zwabs * powerlaw if the redsift is available) in XSPEC and get the simulated fluxes and spectral indices of the $n$ slices. Here the column densities of both the Milk Way and the host galaxy are fixed to the obsered values as in Step 1.

(5) We compare the simulated fluxes and spectral indices with the observed ones and access the goodness of the fits using $\chi^{2}$ statistics.

(6) We refine the trial set of parameters based on the comparison and repeat steps (2)(5) when necessary. We test whether we can reach a set of best-fitting parameters that can reproduce both the light curve and the apparent spectral evolution as observed.

\section{An example: GRB050814}

We apply the method to GRB050814, a typical burst with well-observed X-ray tail with strong spectral evolution. As seen in Fig.2, the tail has a steep decay index of $\sim 3.2$, and a strong spectral evolution is apparent at $t<600 \mathrm{~s}$. These features are common in most

\footnotetext{
${ }^{3}$ Notice that $k$ is fixed to a certain value for a particular model, and is varied when different models are explored.

${ }^{4}$ The PC mode spectra become harder at the end of this tail, which might be due the contamination of the harder shallow decay component. For simplification, we focus on the WT mode data only.
} 
of the GRB X-ray tails. We first fix $k=1$ in Eq. 5, which corresponds to the simplest cutoff powerlaw model. The initial trial parameters we choose are $\left(\Gamma, N_{0, p}, t_{0}, E_{p, 0}, n_{H, h o s t}\right)$ $=(1.2,0.4,72.0,30.0,0.05)$. The peak time $t_{p}$ is fixed to $143.6 \mathrm{~s}$, which corresponds to the end of the prompt emission. Some IDL scripts are developed to follow the procedure described in $\S 3$ to automatically search for the best-fit parameters to match both the observed light curve and the time-dependent spectral index. The final best-fitting parameters are shown in Table 1. The corresponding simulated light curve (black curve) and spectral indices (green curve) are shown in Fig.2. As shown in Fig.2, the sharp decay and the spectral evolution in the tail of GRB 050814 can be indeed explained by the curvature effect with a cutoff power law spectrum.

In order to check the goodness of spectral fit in each time step, we present in Fig.3 the simulated spectra as well as the power fits for each time step. These are compared against the observed time-dependent spectra as well as their power law fits (Fig.4). It can be seen that the model spectra are consistent with the observed ones.

We have also tried other abruptness (the $\mathrm{k}$ parameter) of the spectrum and found that $\mathrm{k}$ cannot be very different from unity. A Band-function spectrum with typical parameters was also tested, but it introduces a less significant spectral evolution that cannot reproduce the data (cf. Qin 2008b).

\section{Discussions and CONCLUSIONS}

We have successfully modeled the lightcurve and spectral evolution of the X-ray tail of GRB050814 using the curvature effect model of a non-power-law spectrum. In particular, a cutoff power law with a standard exponential cutoff $(k=1)$ can well reproduce both the observed lightcurve and time-dependent photon indices. The simulated time-dependent spectra can be well fitted by a power law model with a reasonable $n_{H}$ value. So do the observed time-dependent spectra. This is encouraging, since it has been discussed in the literature (e.g. Fan \& Wei 2005; Barniol-Duran \& Kumar 2008) that the GRB central engine may not die abruptly, and that the observed X-ray tails may reflect the dying history

Table 1: Best-fitting parameters and their 1-sigma errors for the cutoff power curvature effect model for GRB050814. The parameter $k$ is fixed as 1.

\begin{tabular}{lllllll}
\hline$N_{0, p}$ & $E_{c, p}(\mathrm{keV})$ & $\Gamma$ & $t_{0}(\mathrm{~s})$ & $\mathrm{nH}_{\text {host }}$ & $\chi^{2}$ & $\mathrm{dof}$ \\
\hline $0.67(0.12)$ & $10.2(1.3)$ & $1.56(0.25)$ & $103.5(3.4)$ & $0.002(0.04)$ & 10.7 & 9 \\
\hline
\end{tabular}


of the central engine. If this is indeed the case, the strong spectral evolution in the X-ray tails would demand a time-dependent particle acceleration mechanism that gives a progressively soft particle spectrum. Such a behavior has not been predicted by particle acceleration theories. Our results suggest that at least for some tails, the spectral evolution is simply a consequence of the curvature effect: the observer views emission from the progressively higher latitudes from the line of sight, so that the XRT band is sampling the different segments of a curved spectrum. This is a simpler interpretation.

The phenomenology of the X-ray tails are different from case to case (ZLZ07). We have applied our model to some other clean X-Ray tails, such as GRB050724, GRB080523. We find that the data can be interpreted by the model (similar to GRB 050814). Some tails are superposed by X-ray flares, which prevent a robust test of the model. A systematic survey of all the data sample is needed to address what fraction of the bursts can be interpreted in this way or they demand other physically distinct models (e.g. Barniol-Duran \& Kumar 2008; Dado et al. 2008). This is beyond the scope of this Letter.

We thank Pawan Kumar for stimulative discussions and comments. This work is supported by NASA NNG05GB67G, NNX07AJ64G, NNX08AN24G (BBZ\&BZ), by the National Natural Science Foundation of China under Grant 10463001 (EWL) and 10221001 (XYW), and by the National "973" Program of China under Grant 2009CB824800 (EWL\& $\mathrm{XYW})$.

\section{REFERENCES}

Arnaud, K.A., 1996, Astronomical Data Analysis Software and Systems V, eds. Jacoby G. and Barnes J., p17, ASP Conf. Series volume 101.

Barniol Duran, R \& P. Kumar, 2008, arXiv:0806.1226

Band, D et al. 1993, ApJ, 413, 281

Barthelmy, S et al. 2005, Nature, 438, 994

Butler, N. R. \& Kocevki, D. 2007, ApJ, 668, 400

Campana, S. et al. 2006, Nature, 442, 1008

Dado, S., Dar, A., De Rujula, A. 2008, ApJ, 681, 1408

Dermer, C. D. 2004, ApJ, 614, 284

Dyks, J., Zhang, B. \& Fan, Y. Z. 2005 , astro-ph/0511699 
Fan, Y. Z. \& Wei, D. M. 2005, MNRAS, 364, L42

Fenimore, E. E., Madras, C. D., \& Nayakshin, S. 1996, ApJ, 473, 998

Gehrels, N., et al. 2004, ApJ, 611, 1005

Kumar, P. \& Panaitescu, A. 2000, ApJ, 541, L51

Liang, E. W., Zhang, B., O’Brien, P. T., Willingale, R., et al. 2006, ApJ, 646, 351

Liang, E.-W., Zhang, B.-B., Zhang, B., 2007. ApJ 670, 565.

Mészáros, P. 2006, Rep. Prog. Phys. 69, 2259

Nousek, J. A., Kouveliotou, C., Grupe, D., Page, K. L., et al. 2006, ApJ, 642, 389

O’Brien, P. T., Willingale, R., Osborne, J., Goad, M. R., et al. 2006, ApJ, 647,1213

Qin, Y.-P. 2008a, ApJ, in press (arXiv:0804.2175)

Qin, Y.-P. 2008b, ApJ, submitted (arXiv:0806.3339)

Rossi, E., Lazzati, D., \& Rees, M. J. 2002, MNRAS, 332, 945

Starling, R. L. C. et al. 2008, MNRAS, 384, 504

Tagliaferri, G., Goad, M., Chincarini, G., Moretti, A., et al. 2005, Nature, 436, 985

Wu, X. F. et al. 2006, 36th COSPAR Sci. Ass. \#731 (astro-ph/0512555)

Yonetoku, D. et al. 2008, PASJ, 60, S352

Zhang, B. 2007, ChJAA, 7, 1

Zhang, B., Fan, Y. Z, Dyks, J., Kobayashi, S., et al. 2006, ApJ, 642, 354

Zhang, B. \& Mészáros, P. 2002, ApJ, 571, 876

Zhang, B.-B., Liang, E.-W., Zhang, B., 2007, ApJ 666, 1002 (ZLZ07) 